\title{
Fabrication of a coupler waveguide cavity based on large-scale sacrificial layer technology
}

\author{
Xueli Nan, Binzhen Zhang, Xing Yang, Jianli Cui, Shaolei Ge \\ Science and Technology of Electronic Tests \& Measurements Laboratory, North University of China, \\ Taiyuan 030051, China
}

Keywords: Sacrificial layer; Large-scale; PDMS; 3D microwave structure; directional coupler

\begin{abstract}
To solve the difficult problem for the removal of large size sacrificial layers when preparing a terahertz coupler, a new approach is proposed using poly two methyl silicone as a sacrificial layer. Due to its hydrophobicity and high elasticity, cross-linked polydimethylsiloxane (PDMS) can be gently withdrawn from the cavity just after the upper suspension structure is completed. To achieve a robust super structure, we spirt silicon dioxide on the surface of the PDMS to increase its hydrophilicity resulting in large scale preparation of the sacrificial layer. This process greatly reduces the steps and equipments needed for traditional processing technologies. To obtain a good suspended structure, we sputtered silica on its surface to increase the hydrophilicity of PDMS. Experiments show that PDMS may fill the entire cavity, and that the surface has a certain adhesion after treatment, satisfying the preparation requirements of the upper structure. This approach solves the problems involving the fabrication process such as difficulty in removing the large-scale sacrificial layer, reducing the number of complex microfabrication steps and the equipment required resulting in a more cost and time - effective solution. The duration for removal of PDMS from SU-8 structures is also much lower (saving approximately 95\% of time) compared to conventional photoresistance technology.
\end{abstract}

\section{Introduction}

Terahertz science and technology has made rapid developments over the past two decades and now has great potential in the application of high frequency wireless communications especially in the military field ${ }^{[1]}$. Currently, with the advanced MEMS technologies ${ }^{[2]}$, microsensors ${ }^{[3]}$ and microcouplers have been used in many industrial fields ${ }^{[4,5]}$.

The design and fabrication of low cost terahertz devices are increasing the range of potential applications including areas of science, security ${ }^{[3]}$, communication ${ }^{[4]}$, imaging ${ }^{[5]}$, environment and manufacturing $^{[6]}$ and biomedicine ${ }^{[7]}$. A sacrificial layer is a special kind of material that is used to improve the preparation of the suspended structure. The function of the sacrificial layer is to provide strong mechanical strength to support the upper structure that can be easily removed. The sacrificial layer material has been reported mainly to contain monocrystalline silicon, porous silicon (PS), silica and the photosensitive resist ${ }^{[8]}$. Currently, the best solution is to use photosensitive resist as a sacrificial layer, such as SU-8, PMMA and polyimide ${ }^{[9]}$. However, due to the high temperature treatment, SU-8 and PMMA are more difficult to remove in a large-scale three-dimensional cavity and the processing time is relatively long. To meet the complex requirements of internal cavities, there is an urgent need to find a new sacrificial layer material which is easy to fill the complex cavity and also easy to remove. In addition, due to the complex requirements of internal cavity shape, the material must also be able to fill cavity structures of complex shape.

In this paper, we used PDMS to generate a large-scale sacrificial layer in the fabrication of a straight waveguide cavity ${ }^{[0]}$. In view of its unique hydrophobicity, the layer does not react with other substances after it is cured and is easy to remove. Compared to SU-8 ${ }^{[11]}$, PMMA and other photosensitive resists, the production process for PDMS is fast, simple, and cross linked PDMS can be gently withdrawn from the cavities after the upper suspended structure is completed. This approach not only solves the problem of high cost associated with the sacrificial layer but also simplifies the fabrication process including prebake, exposure, development, resist removal and 
micro electroforming ${ }^{[12,13]}$ : By simplifying the production process, production efficiency and speeds improve the production rate. More importantly, PDMS does not have the internal stress which exists in a general photosensitive sacrificial layer ${ }^{[14]}$ and can be used to obtain a high accuracy cavity due to its high shifting precision.

\section{Experiments}

This study focused on the preparation process of a straight waveguide cavity. The preparation procedure consists of three parts; the first part uses a UV thick photosensitive resist SU-8 to fabricate cavity walls; the second part is to fill the entire cavity with PDMS which is then heated on a dry counter until cured; the third part is to release the sacrificial layer. The quality of the sacrificial layer directly determines the preparation of superstructure. A detailed schematic of the fabrication process of straight waveguide cavity is shown in Fig. 1.

\section{\\\\}}

(a)

(b)

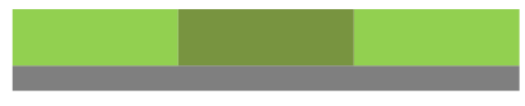

(c)

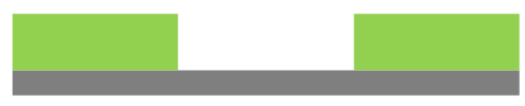

(d)

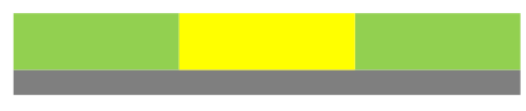

(e)
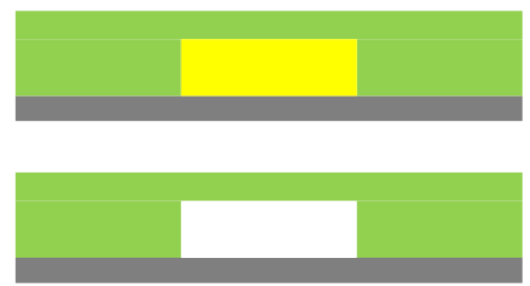

Si

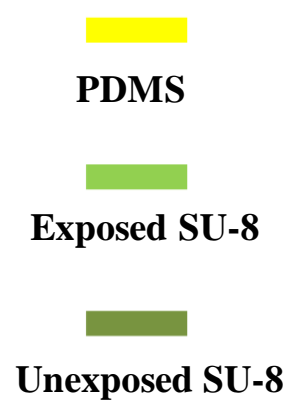

Fig.1 Schematic diagram of the fabrication process for a straight waveguide cavity

First, SU-8 resistance was used to prepare the sidewall structure. Due to its low thermal expansion coefficient and thermal conductivity, this results in straight sidewall profiles and can be used for manufacturing of a multilayer structure. All the fabrication steps were carried out in a class 100 lithography clean room. The UV- LIGA technology process of SU-8 adopted in this paper is similar to that reported by Yang and Wang $(2005,2004)$, and Shao et al.(2012,2010) ${ }^{[15]}$. To obtain a Large-scale straight waveguide cavity with a length of $23 \mathrm{~mm}$, the total height is $300 \mathrm{um}$. The treated SU-8 mold was then ready to be utilized in the following process (Fig. 1a 1b).

A PDMS polymer was prepared with a Sylgard184 prepolymer with 15:1 - a weight ratio of the prepolymer to curing agent. The prepared polymer was then degassed in a vacuum desiccator to remove air bubbles after being thoroughly mixed. Once all the bubbles have cleared from the mixture (now we will refer to the mixture as PDMS), a pipette was used to carefully coat the desired cavities of the sample mold of the SU-8. The sample mold was filled to the top of the cavity to ensure an even surface. Thirdly, the PDMS mold was carefully placed onto the hotplate, and the samples were baked for 2-15 minutes. Whether or not the samples are solidified is checked by pressing it using toothpicks. If the samples were solidified, they were taken out. Baking time and temperature were adjusted continuously so as to ensure that no bubbles are expanded in the PDMS. If bubbles were produced, the bubbles dried resulted in a hole in the PDMS mold. PDMS could dry on its own within about 24 hours at room temperature, so baking PDMS at lower temperature for a longer time might be the best way to ensure that no bubbles in the PDMS were expanded. In this research, the mold was baked at $100^{\circ} \mathrm{C}$. The cross section of a T-shaped cantilever on the microchip was shown in Fig. 1c. 
Surface treatment of PDMS is necessary to better prepare for the suspended structure, a thin layer of silicon dioxide was sputtered onto the surface after plasma treatment. The silicon dioxide layers with different thicknesses with varying sputtering times were obtained. And then SU-8 photosensitive resist was coated onto the sputtered surface (Fig. 1d).Finally, the cross-linked PDMS was gently withdrawn from the cavity. The removed cured PDMS mold down from the hotplate and put them in a freezer for a few minutes; this will shrink the PDMS slightly and help to peel the samples delicately out of their molds (Fig. 1e).

\section{Result and discussion}

\section{Three dimensional morphology of waveguide cavity}

A silica layer was sputtered onto the surface of the completed structure and then the structure observed using a scanning electron microscope(SEM). In this research, the coupler with a T-type waveguide cavity structure has large length, therefore SEM is only able to capture its local structure. The three-dimensional micrographs scanned using SEM for each section of the waveguide cavity are shown in Figure 2.

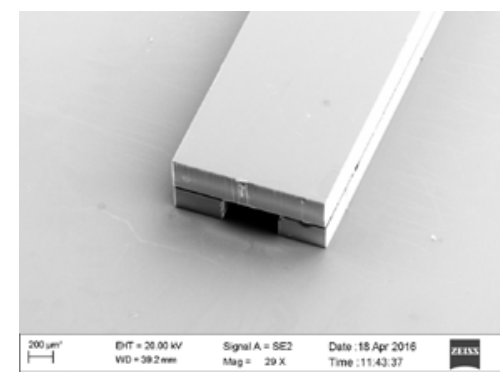

a.waveguide cavity

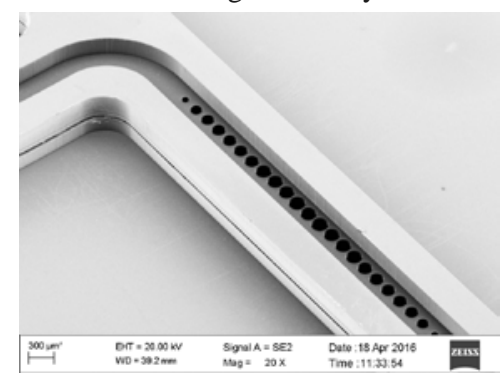

c. coupler hole

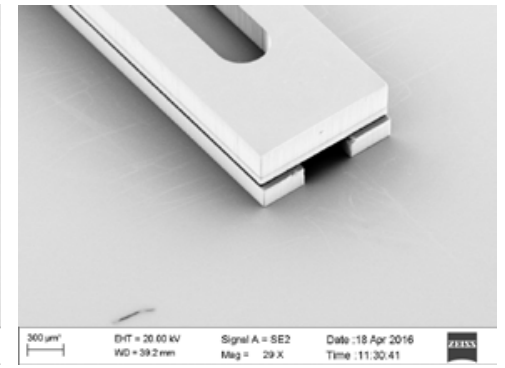

b.upper coupler cavity

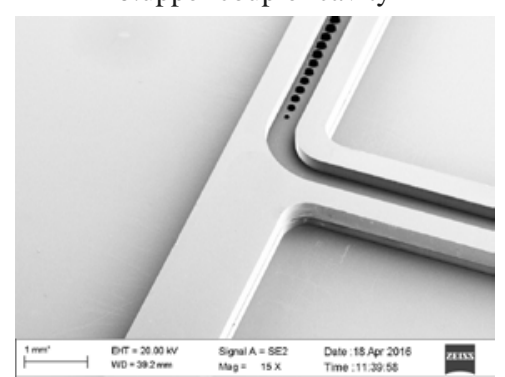

d. integrated structure

Fig2. SEM photograph of the Coupler waveguide cavity: a Straight waveguide cavity structure after the sacrificial layer is released; b Partial view of its upper coupler cavity; c Enlarged view of the upper coupler hole;

$\mathrm{d}$ Integral structure of the coupling cavity and the coupling hole

From Fig. 2a and 2b, it is found that the complete structure of the waveguide cavity can be obtained by using the sacrificial layer technology. The process of removing large scale sacrificial layers is simple and fast. After the sacrificial layer was released, the integrate structure of good side walls was obtained. Fig. 2c and 2d are the local structures of coupling holes prepared on the sacrificial layer. At the smallest coupling aperture diameter size of $0.24 \mathrm{~nm}$, the structure of the coupling hole is good, being fully developed. It can also be seen that using this sacrificial layer technology to process the cavity and the complex supper structure is feasible.

\section{Contact angle test}

Hydrophobicity means that PDMS does not easily adhere to other substances. At the same time, the hydrophobicity leads to difficulties when constructing a new suspension structure on the surface of the PDMS. A hydrophilic modification was favorably conducted on the surface of the PDMS by coating with the SU-8. Prior to the spin coating of SU-8, we treated the cured PDMS with plasma to improve the surface wettability. The curve of the contact angle with the plasma processing time is shown in Figure 4. 


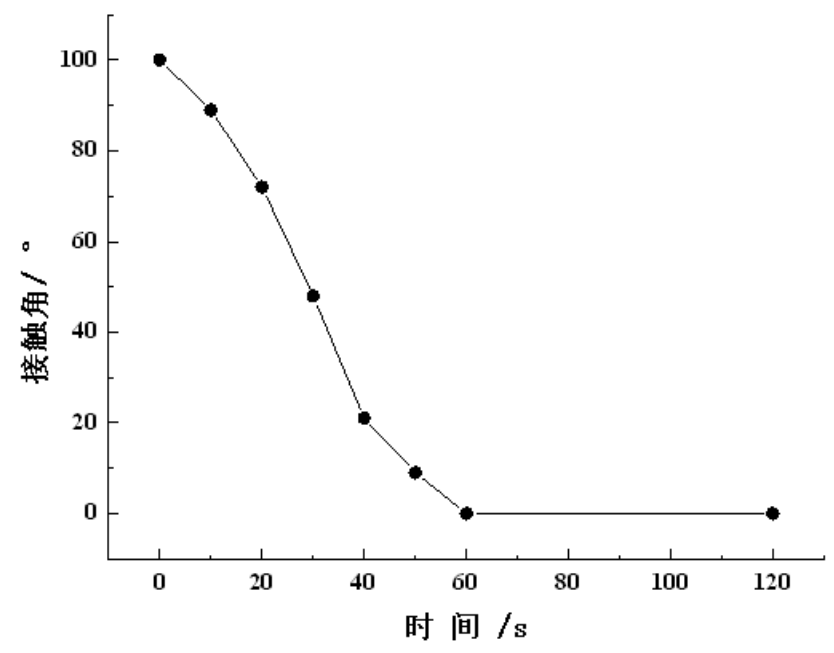

Figure 3. The curve of the contact angle with plasma treatment time

The data curve of Figure 3 shows that the contact angle of PDMS surface decreases rapidly with the increase of plasma treatment time. The surface contact angle of the untreated PDMS is about 100 degrees which shows that the surface wettability of PDMS is very poor.

Due to the PDMS formula having no hydroxyl, carboxyl and other hydrophilic function groups, there is no direct interaction being happened between PDMS molecules and water molecules. The photosensitive resist is therefore difficult to adhere to the surface of the PDMS, which results in a small contact surface and non-uniform spin coating . As a result, the preparation of the preparation of the superstructure is influenced.

Along with the increase of surface treatment time, the contact angle decreases. When the plasma treatment time continues for 60s, the PDMS surface contact angle reached $0^{\circ}$ (droplets completely spread out) and so the contact angle will no longer change.

\section{Surface roughness measurements of the sacrificial layer}

The surface morphology and roughness of the modified PDMS were observed by atomic force microscope (AFM).Comparison of surface roughness of the modified PDMS is show in Figure 4.
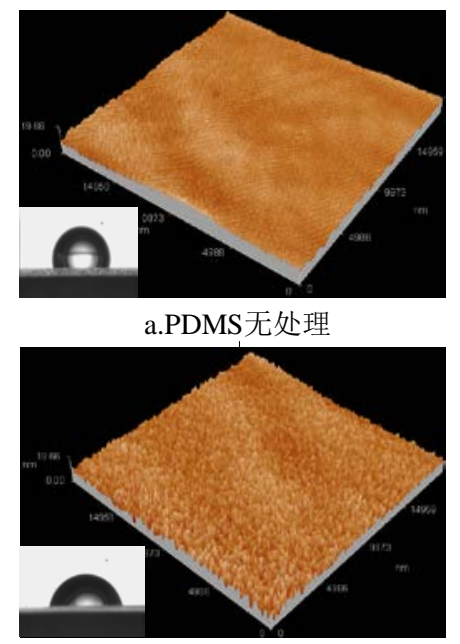

c.plasma处理20s

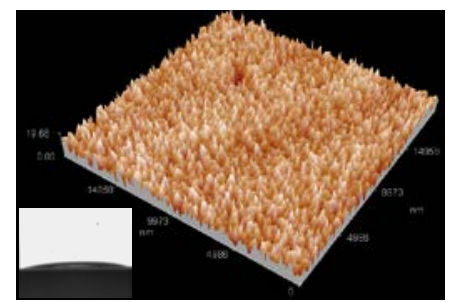

b.plasma处理60s

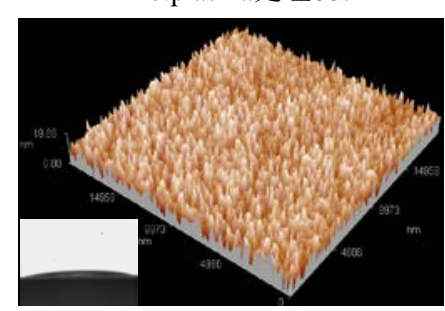

d.plasma修饰20s, 溅射 $20 \mathrm{nmSiO} 2$

Figure 4. Surface morphology test of the sacrificial layer after treatment

Fig 4a represents the 3D graphics and contact angle test of the PDMS surface without any modification, showing the PDMS surface is flat and level, with no projections or subsidence and the roughness is $0.88 \mathrm{~nm}$ with a contact angle around $100^{\circ}$. Fig $4 \mathrm{~b}$ represents the three-dimensional view and a contact angle view of the PDMS surface topography after plasma modification for 60 seconds. After a long time of plasma modification, the PDMS surface produced a large number of bumps and burr as the surface morphology changed greatly. The roughness became $3.44 \mathrm{~nm}$ and the 
contact angle was close to $0^{\circ}$. Fig 4c shows the 3D graphics and contact angle test of the PDMS surface after plasma modified for 20 seconds. After a short time of plasma treatment, the sample surface appears to have a significant uplift. However, the average height of the uplift is relatively low and roughness increases from $0.88 \mathrm{~nm}$ to $1.24 \mathrm{~nm}$ with a contact angle that has a greater degree of reduction from $100^{\circ}$ to about $70^{\circ}$. A surface morphology test was conducted by sputtering $20 \mathrm{~nm}$ thick silica on the PDMS surface. The results obtained by the treatment within $15 \mathrm{~s}$ in the test are shown in Fig. 4d. It can be seen that the surface roughness has become $3.51 \mathrm{~nm}$, which is greater than that of in Fig.4b, while the contact angle has achieved the same level as that of Fig.4b.

In summary, when the combined modification method and a single plasma modification method achieve the same treatment results, the combined modification method requires less time for plasma pre-treatment. Afterwards, there is only a simple secondary modification using surfactant to be needed; optical microscope observations result show that the combined modification method will cause no mechanical damage to PDMS, which ensures that the modified PDMS as a good support structure can be used to prepare favorable suspended structures.

\section{Conclusion}

A large scale three-dimensional waveguide cavity structure (23 mm x $280 \mathrm{um}$ ) was successfully fabricated using PDMS as a sacrificial layer. The cross-linked PDMS was gently extracted from the prepared cavity structure just after the suspension of the structure was accomplished. This process greatly simplified the process and equipments used in traditional processing technologies, the durations for removing PDMS from SU-8 structures are smaller, which approximately saves 95\% time compared to conventional photoresist technologies. More importantly, there is no internal stress in PDMS which exists in regular photosensitive sacrificial layer, and owing to the high transfer accuracy, and ease of removal, high accuracy complex cavity structure can be obtained. Due to its low cost, non-toxic and pollution-free, the method greatly reduces the cost of fabrication and is both safe and environmentally friendly. This new type of sacrificial layer technology simplifies the production process, improves the processing speed and the complex multi-layer cavity structures prepared has wide ranging potential applications.

\section{Acknowledgments}

This research is supported by the National Natural Science Foundation of China (Grant No.51475438). The experimental work was conducted at the State Science and Technology on Electronic Tests \& Measurements Laboratory of The North University of China and The State Key Laboratory for Manufacturing System Engineering of Xi'an Jiao tong University of China.

\section{References}

[1] RAMADAN K S, NASR T, FOULDS I G. Development of an SU-8 MEMS process with two metal electrodes using amorphous silicon as a sacrificial material [J]. Journal of Micromechanics and Microengineering, 2013, 23(3): 035037.

[2] CAI H, DING G, YANG Z, et al. Design, simulation and fabrication of a novel contact-enhanced MEMS inertial switch with a movable contact point [J]. Journal of Micromechanics and Microengineering, 2008, 18(11): 115033.

[3] ABGRALL P, CONEDERA V, CAMON H, et al. SU-8 as a structural material for labs-on-chips and microelectromechanical systems [J]. Electrophoresis, 2007, 28(24): 4539-51.

[4] AMARI S, ROSENBERG U, BORNEMANN J. Adaptive synthesis and design of resonator filters with source/load-multiresonator coupling [J]. Microwave Theory and Techniques, IEEE Transactions on, 2002, 50(8): 1969-78.

[5] BAO X, DARGENT T, CATTAN E. Micromachining SU-8 pivot structures using AZ 
photoresist as direct sacrificial layers for a large wing displacement [J]. Journal of Micromechanics and Microengineering, 2010, 20(2): 025005.

[6] LAU K H, GIRIDHAR A, HARIKRISHNAN S, et al. Releasing high aspect ratio SU-8 microstructures using AZ photoresist as a sacrificial layer on metallized Si substrates [J]. Microsystem technologies, 2013, 19(11): 1863-71.

[7] LEE J I, SHIKIDA M, SATO K. Polymer micromachining technologies for a table-shaped tactile sensor using thick sacrificial layer [J]. Microsystem Technologies, 2015, 21(1): 239-46.

[8] YANG X, FAN Y, ZHANG B, et al. A Novel Terahertz Rat-Race Hybrid Coupler Based on PPDW [J]. Journal of Infrared, Millimeter, and Terahertz Waves, 2011, 32(11): 1291-8.

[9] ZAHRILADHA Z, SAM W Y, ABIDIN M Z, et al. The integration of rectangular SIW filter and microstrip patch antenna based on cascaded approach [M]. Malaysian Technical Universities Network (MTUN). 2012.

[10]YANG J, SHI Y, ZHONG H, et al. Preparation of sacrificial layer for MEMS devices by lift-off technology [J]. Microsystem Technologies, 2014, 20(2): 259-63.

[11] YANG L, HAO X, WANG C, et al. Rapid and low cost replication of complex microfluidic structures with PDMS double casting technology [J]. Microsystem Technologies, 2014, 20(10): 1933-40.

[12]LEE J-W, SHARMA J, NARDUCCI M S, et al. Cavity-enhanced sacrificial layer micromachining for faster release of thin film encapsulated MEMS [J]. Journal of Micromechanics and Microengineering, 2015, 25(6): 065010.

[13]LIU J, ZHANG D, SHA B, et al. Fabrication of a three-layer SU-8 mould with inverted T-shaped cavities based on a sacrificial photoresist layer technique [J]. Biomedical microdevices, 2014, 16(5): 655-60.

[14]LUCIBELLO A, PROIETTI E, MARCELLI R, et al. Smoothing and surface planarization of sacrificial layers in MEMS technology [J]. Microsystem technologies, 2013, 19(6): 845-51.

[15]SHAO G, QIU W, WANG W. Fast replication of out-of-plane microlens with polydimethylsiloxane and curable polymer (NOA73) [J]. Microsystem Technologies, 2010, 16(8): 1471-7. 
